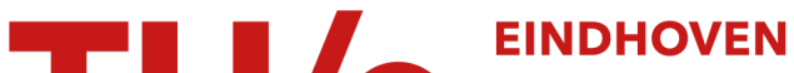 UNIVERSITY OF TECHNOLOGY
}

\section{Recent research advancements in in-building optical networks}

Citation for published version (APA):

Koonen, A. M. J., Okonkwo, C. M., Shi, Y., Boom, van den, H. P. A., Tran, N. C., \& Tangdiongga, E. (2011). Recent research advancements in in-building optical networks. In Proceedings of the 13th International Conference on Transparent Optical Networks (ICTON), 26-30 June 2011, Stockholm, Sweden (pp. Tu.A5.1-1/5). Institute of Electrical and Electronics Engineers. https://doi.org/10.1109/IĆTON.2011.5970901

DOI:

10.1109/ICTON.2011.5970901

Document status and date:

Published: 01/01/2011

\section{Document Version:}

Publisher's PDF, also known as Version of Record (includes final page, issue and volume numbers)

\section{Please check the document version of this publication:}

- A submitted manuscript is the version of the article upon submission and before peer-review. There can be important differences between the submitted version and the official published version of record. People interested in the research are advised to contact the author for the final version of the publication, or visit the $\mathrm{DOI}$ to the publisher's website.

- The final author version and the galley proof are versions of the publication after peer review.

- The final published version features the final layout of the paper including the volume, issue and page numbers.

Link to publication

\section{General rights}

Copyright and moral rights for the publications made accessible in the public portal are retained by the authors and/or other copyright owners and it is a condition of accessing publications that users recognise and abide by the legal requirements associated with these rights.

- Users may download and print one copy of any publication from the public portal for the purpose of private study or research.

- You may not further distribute the material or use it for any profit-making activity or commercial gain

- You may freely distribute the URL identifying the publication in the public portal.

If the publication is distributed under the terms of Article $25 \mathrm{fa}$ of the Dutch Copyright Act, indicated by the "Taverne" license above, please follow below link for the End User Agreement:

www.tue.nl/taverne

Take down policy

If you believe that this document breaches copyright please contact us at:

openaccess@tue.nl

providing details and we will investigate your claim. 


\title{
Recent Research Advancements in In-Building Optical Networks
}

\author{
Ton Koonen, Chigo Okonkwo, Yan Shi, Henrie van den Boom, Nguyen-Cac Tran, Eduward Tangdiongga \\ COBRA Institute, Eindhoven Univ. of Technology, \\ P.O. Box 513, NL 5600MB Eindhoven, The Netherlands \\ e-mail:a.m.j.koonen@tue.nl
}

\begin{abstract}
For in-building communication, a powerful backbone network can be created by means of optical fibre, which in a single infrastructure can offer a wealth of both fixed and wireless service connections to the users. Next to the technological challenges, important aspects to be taken into account are the economics of network installation and operation, and sustainability. An overview is given of recent research results regarding the optimization of network architectures, and regarding advanced signal processing for economical and sustainable delivery of high-capacity wired and wireless services over various types of multimode (plastic) optical fibre.
\end{abstract}

Keywords: in-building network, techno-economics, plastic optical fibre, radio-over-fibre, wavelength routing.

\section{INTRODUCTION}

To meet the rapid growth of bandwidth demand from the users, driven by the exploding gamma of internet services, video services, fast peer-to-peer file exchange, etc. etc., the roll-out of fibre-to-the-home networks is not sufficient [1] ; the information highway needs to be extended beyond the doorstep of the user's home, into the home itself, up to his communication devices. The communication traffic inside the home may ask for even higher bandwidths, such as for fast transfer of large files to and from data servers. Presently, there is a mixture of separate networks in the residential home, each optimised for delivery of a specific set of services: twisted pair for telephony services, coaxial cable for TV and FM radio broadcast services, Cat-5E cables and WiFi for computer data services, and possibly other networks for other home appliances such as controlling the central heating, burglar alarms, door bell, etc. A single network in which the delivery of all services is integrated offers many advantages: easier installation, easier maintenance, easier upgrading and introduction of new services, facilitating communication between devices with different network interfaces, etc. The signal processing, storage, translation and intelligent routing functions can be handled in a centralised module, the residential gateway $(\mathrm{RG})$, which also provides the interfacing with the access network, and takes care of other tasks such as assuring security and privacy. Optical fibre is the ideal transport medium for such an integrated in -home backbone network. Silica multimode fibre (MMF) and single-mode fibre (SMF) have excellent characteristics, but are fragile and need careful handling during installation. Plastic optical fibre (POF) is easier to install due to its ductility, and especially large-core POF reduces installation costs as it is very easy to couple ('do-it-yourself').

In this paper, we will report about recent studies on the cost analysis of in-building networks, comparing different network topologies and cable media choices and addressing both network installation costs (CapEx, capital expenditures) and network operation costs (OpEx, operational expenditures). We will also discuss advanced signal routing functions to improve the utilisation of the network's resources and to reduce the energy consumption.

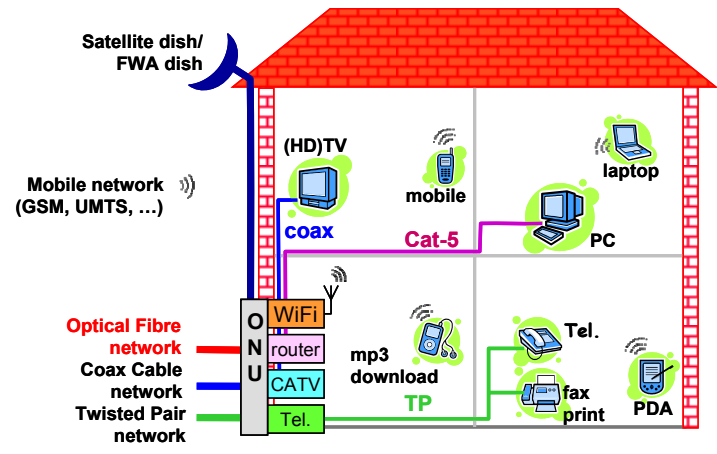

a) separate networks

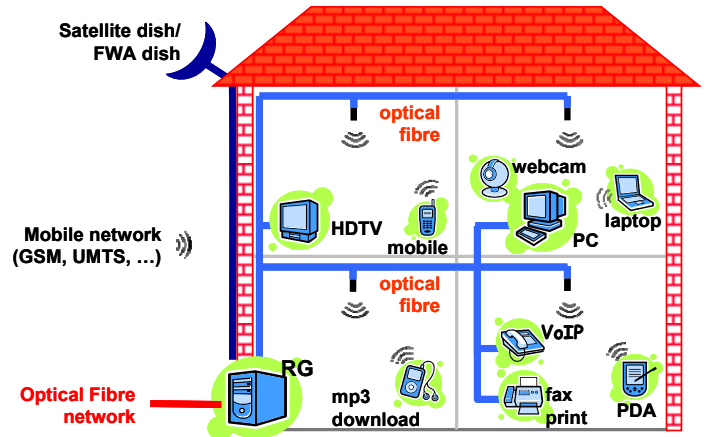

b) integrated network

Figure 1. Service provisioning networks in the home.

\section{OPTIMIZING IN-BUILDING NETWORK ARCHITECTURES}

Depending on the layout of a building, a wide variety of network topologies may be considered. For our costs analysis, we have considered the basic topologies shown in Figure 2: a point-to-point (P2P) topology, point-tomultipoint (P2MP) bus and tree topologies, and hybrid topologies such as star-tree (which enable coverage of 
a larger building given the restrictions on the individual link length due to the cable medium properties). Table 1 lists the typical cost and power consumption data of the network items, which we have used to estimate the CapEx and OpEx of these topologies. We have assumed an opaque network architecture carrying gigabit Ethernet traffic, so a network with active opto-electronic-optical signal conversions in the nodes (e.g. for IP packet routing). The details of our analysis method have been reported in [2]. We have analysed three typical building scenarios: a residential home (single family house, with 3 floors and 4 rooms per floor), office building (10 floors, 50 office rooms per floor), and a multi-dwelling units (MDU) building (apartment building, with 10 floors and 16 living units (LUs) per floor). As reported in [3], we found that for smaller buildings (such as a residential home) a P2P topology has the lowest network installation costs. When large-core (1mm diameter) duplex POF cabling is used, the CapEx per room is only slightly higher than when Cat-5E cabling is used. When the duplex POF cables are put in the ducts which are already hosting the electrical power cables, duct costs are saved and the duplex POF P2P network solution is already cheaper than the Cat-5E one, notwithstanding the fact that the market volume of POF is still ramping up. For larger buildings (such as an MDU or office building), we found that the CapEx is lowest when a bus topology is adopted.

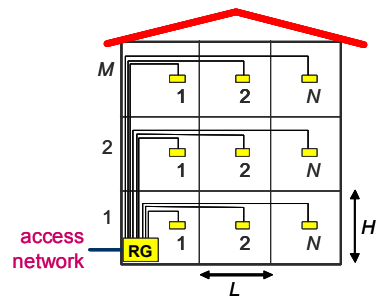

a) point-to-point $(\mathrm{P} 2 \mathrm{P})$

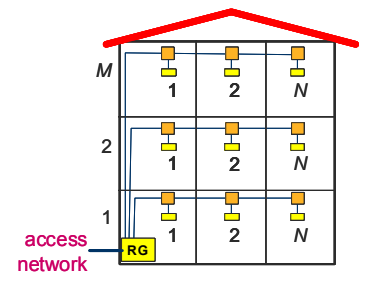

b) bus

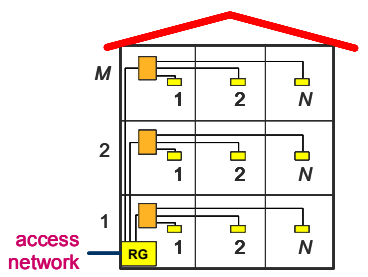

c) tree

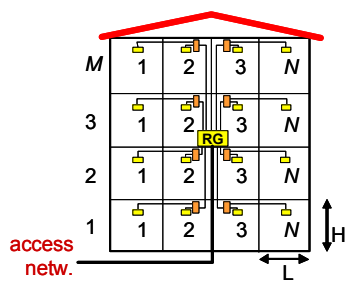

d) star-tree

Figure 2. In-building network topologies

Table 1. Costs of network items and their power consumption.

\begin{tabular}{|l|c|c|c|c|}
\hline & Cat-5E & POF & SMF & MMF \\
\hline Installed cable costs & $1.8 € / \mathrm{m}$ & $1.7 € / \mathrm{m}$ & $1.74 € / \mathrm{m}$ & $1.95 € / \mathrm{m}$ \\
\hline Max. link length & $100 \mathrm{~m}$ & $70 \mathrm{~m}$ & $1000 \mathrm{~m}$ & $550 \mathrm{~m}$ \\
\hline Mounted connector costs & $13 €$ & $3 €$ & $15 €$ & $14 €$ \\
\hline Media converter costs; power cons. & negligible; $0.65 \mathrm{~W}$ & $30 € ; 0,85 \mathrm{~W}$ & $70 € ; 1.15 \mathrm{~W}$ & $40 € ; 1.15 \mathrm{~W}$ \\
\hline Hub/tap costs; power consumption & $20 € ; 0.2 \mathrm{~W}$ & $20 € ; 0.2 \mathrm{~W}$ & $20 € ; 0.2 \mathrm{~W}$ & $20 € ; 0.2 \mathrm{~W}$ \\
\hline Switch costs, power consumption & $10 € /$ port; & $10 € /$ port; & $10 € /$ port; & $10 € /$ port; \\
& $0.3 \mathrm{~W} /$ port & $0.3 \mathrm{~W} /$ port & $0.3 \mathrm{~W} /$ port & $0.3 \mathrm{~W} /$ port \\
\hline
\end{tabular}

Figure 3a shows the network installation costs per LU when typical dimensions are assumed for the MDU building (10 floors, 16 LUs per floor, LU height $4 \mathrm{~m}$, link length $14 \mathrm{~m}$ between outlets in neighbouring LUs). The installation costs of the duplex POF solution are clearly the lowest, where the cost advantage with respect to the Cat-5E solution is mainly due to duct sharing with the electricity wiring. The media converters (optoelectronic transceivers in the active bus network nodes) still contribute strongly to the installation costs; when the POF transceiver market gets larger and the products more mature the media converter costs are expected to come down remarkably.

Figure $3 \mathrm{~b}$ shows the power consumption per LU in the MDU building, which is a major contributor to the OpEx per LU. As the Cat-5E bus network does not need media converters in the bus nodes, the power consumption of the Cat-5E solution is the lowest; the power consumption of the duplex POF solution is only slightly higher.

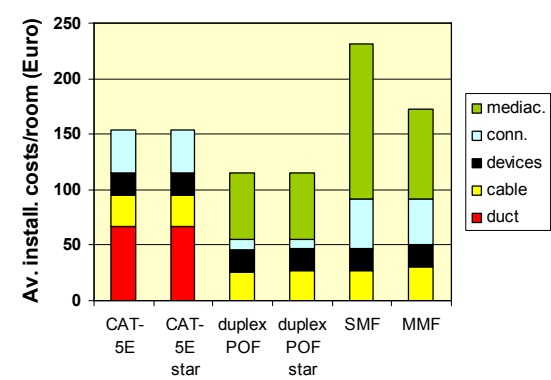

a) installation costs per LU (with duct sharing for the fibre-based solutions)

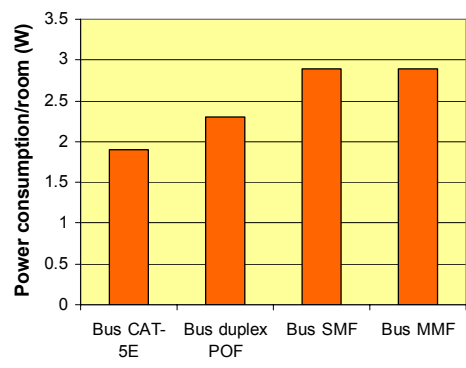

b) Power consumption per LU

Figure 3. Installation costs and power consumption per living unit of a bus network in a multi-dwelling units building (10 floors, 16 living units per floor). 
When deciding which cable medium to take, one must consider the total costs of a network solution, so the CapEx plus the OpEx during the network's lifetime. These are dependent on future price evolutions of the CapEx and OpEx items. As a first estimate, the total costs of the duplex POF bus network solution for an MDU building appear to be lower than those of the Cat-5E solution.

\section{DYNAMIC RECONFIGURATION OF THE IN-BUILDING NETWORK}

The efficiency of service provisioning in a large in-building network can be considerably improved when the available capacity is not assigned statically to all the rooms in the building, but when it is dynamically routed to those places where there is an actual traffic demand. In particular for wireless services, the local traffic demand may fluctuate considerably when users roam throughout the building (e.g. students moving from one classroom to an other one).

The dynamic routing of traffic streams may be implemented by using wavelength multiplexing of these streams and wavelength routing in the network nodes. Using our optical frequency multiplying technique [4] , we can efficiently generate $\mathrm{mm}$-wave radio signals remotely while only a moderate sweep frequency signal is needed at a centralized location in the building (i.e. the residential gateway). As shown in Figure 4, at the RG the radio data signal (generated by the vector signal generator, at 120Mbit/s in a 64-QAM format on an IF carrier frequency of $3.6 \mathrm{GHz}$ ) is intensity-modulated with negligible chirp on a specific CW wavelength channel, and subsequently phase-modulated by a sinusoidal sweep signal of $6 \mathrm{GHz}$ [5] . After transversing an optical amplifier (EDFA) and an SMF link, a microring resonator (MRR) filter tuned to the wavelength channel acts as an adddrop multiplexer and drops the wavelength channel to a local photodiode. When operating on the filter slope of the MRR, the phase modulation impressed on the wavelength channel is converted to intensity modulation. As this is a heavily non-linear process, many harmonics of the $6 \mathrm{GHz}$ sweep frequency are generated. Selecting the $6^{\text {th }}$ harmonic by means of an electrical bandpass filter after photodetection yields the desired $39.6 \mathrm{GHz} \mathrm{mm}$ wave radio signal. Thus we demonstrated the remote generation and wavelength-selective routing of an 39.6 GHz $120 \mathrm{Mbit} / \mathrm{s}$ 64-QAM radio signal, with an error vector magnitude of less than 5\%.

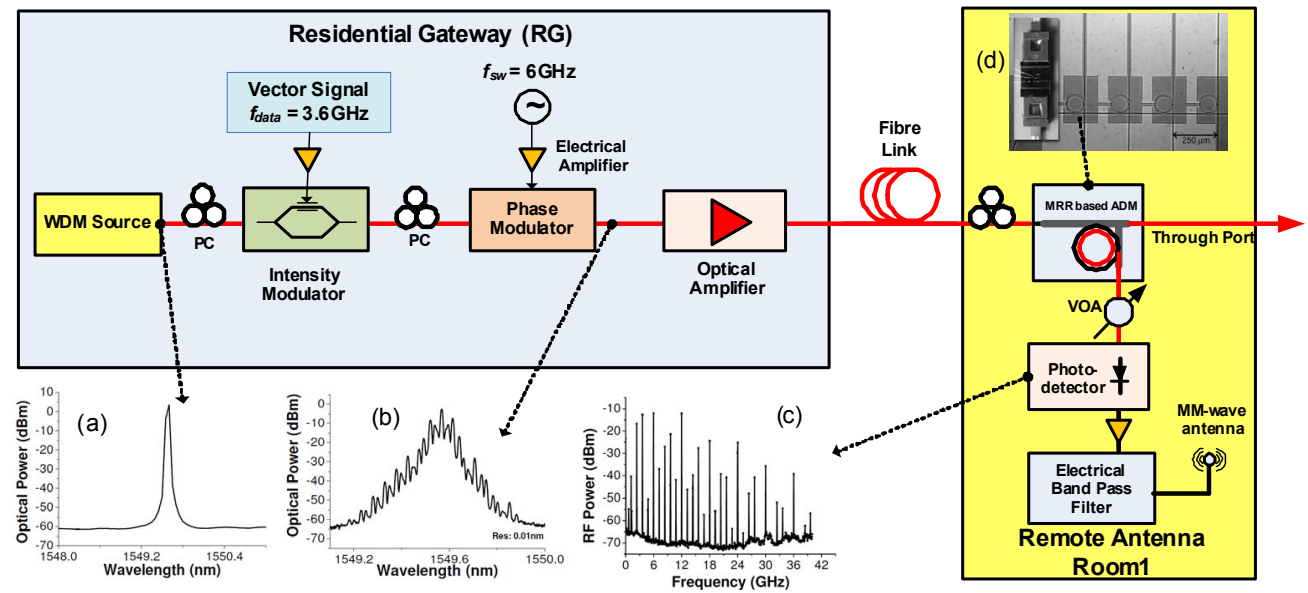

Figure 4. Micro-ring assisted generation and routing of an mm-wave radio signal.

We have analysed how the actual network performance can be improved with wavelength routing techniques [6] . The network performance has been measured by means of the network congestion probability, i.e. the probability that anywhere in the network a call is blocked due to a lack of capacity in a wavelength channel. We have analytically modelled the traffic statistics per LU as an on-off process, where the LU needs a capacity $R$ when active. We assumed $W$ wavelength channels, each with a data transport capacity $B$ (so the total network capacity is $W \cdot B$ ). We also assume that the LU-s behave independently, and that the probability of $k$ of the $N$ LU-s being active (with probability $p$ that an individual LU is active) can be modelled with a binomial distribution. By using Chernoff's upper bound, we can derive that

$$
\operatorname{Pr}[k>D] \leq\left(\frac{p}{D}\right)^{D} N^{N}\left(\frac{1-p}{N-D}\right)^{N-D} .
$$

Network congestion in a wavelength channel will occur when $D>B / R$ LU-s want to get access to a wavelength channel. When wavelength routing is applied, a call from an LU may be allocated flexibly to any wavelength channel. Therefore, when a wavelength channel is exhausted, the call may be allocated to a wavelength channel where still capacity is available, and thus the call can still be accepted. Obviously this reallocation process is the most successful when it can be done per individual LU. However, this requires wavelength-routing per LU, which will lead to considerable network complexity and thus higher network costs. The wavelength routing may also be done per cluster of LU-s, as illustrated in Figure 5a. A cluster can be moved to an other wavelength 
channel when needed to avoid congestion, implying that a number of LU-s are together reallocated. The larger the cluster is, the lower the complexity and thus the costs of the network; but larger clusters have also less chance to get successfully reallocated, so the network congestion probability will get worse. Without wavelength routing, the probability of network congestion is

$$
\operatorname{Pr}_{\text {no_reconfig }}(\rho)=1-\left(1-\operatorname{Pr}_{\text {block }}(\rho)\right)^{W}=1-(1-\operatorname{Pr}[k>(B / R)])^{W},
$$

where the average relative network load $\rho=p \cdot N \cdot R /(W \cdot B)$

When wavelength routing can be done per individual LU, and assuming that a reallocated call can fit fully in the other wavelength channel without fragmentation, the probability of network congestion is

$$
\operatorname{Pr}_{\text {reconfig }}(\rho)=\operatorname{Pr}[k>(W \cdot B / R)] .
$$

We have analysed this dynamic network reconfiguration process by means of numerical simulations, for an MDU building with 10 floors and 16 LU-s per floor, so $N=160$ LU-s. We assumed $W=10$ wavelength channels, each with $1 \mathrm{GbE}$ capacity, and also assumed that an LU when active requires a capacity $R=100 \mathrm{MbE}$ (so each wavelength channel can accommodate up to 10 active LU-s, and the whole network up to 100 active LU-s). The results of our analysis are shown in Figure 5b, which shows the network congestion probability versus the relative network load. Curves are given following the analytical equations mentioned above, and following the simulation results for various cluster sizes $c$. The two outermost curves clearly show the impact of wavelength routing: the one to the utmost left depicts the congestion probability without wavelength routing, and the one to the utmost right the congestion probability with wavelength routing per individual LU (so $c=1$ ). For a given acceptable congestion probability of say $10^{-3}$, without routing the allowable network load is only some $25-30 \%$, whereas with finest-grain routing $(c=1)$ the load may go up to $76 \%$. When the cluster size $c$ is increased, the allowable network load clearly becomes less, but this also is favourably accompanied by a lower network complexity. With $c=4$ the allowable network load is still up to $60 \%$, while network complexity is significantly reduced, so for this case $c=4$ seems to be a suitable compromise between network performance and costs.

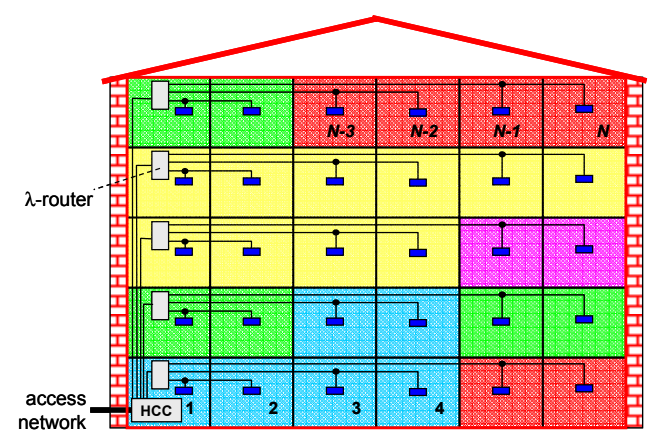

a)

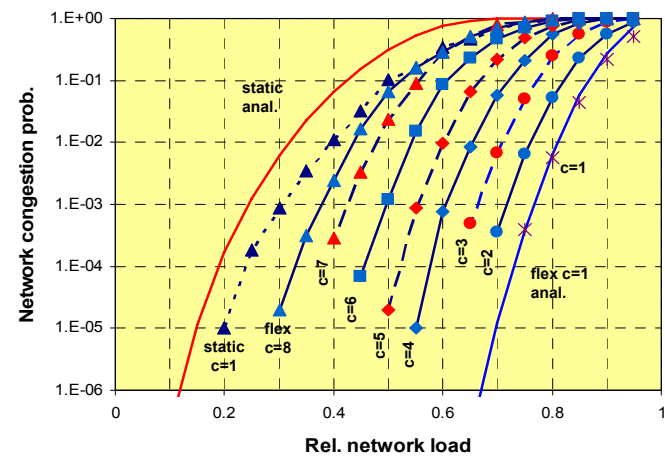

b)

Figure 5. MDU building with wavelength routing; a) routing per cluster of LU-s, with a cluster size of $c=2 \mathrm{LU}-s$; b) network congestion probability without and with wavelength routing for various cluster sizes (markers indicate simulation results).

\section{CONCLUSIONS}

Large-core duplex POF is an attractive medium for low-cost broadband in-building networks. For large buildings such as an MDU, a bus topology using duplex POF and opaque routers in the network nodes can be more cost-efficient than a Cat-5E solution, in particular when duct sharing with the electricity wiring is used. When using SMF, flexible wavelength routing may be employed in larger buildings, and the average network load can be significantly increased. For a typical MDU building, with wavelength routing per cluster of 4 living units, the average network load may roughly be tripled. This implies that less network resources are needed for a certain acceptable call rejection rate, and network installation and energy consumption costs can be reduced.

\section{ACKNOWLEDGEMENTS}

Partial funding of this work by the EC in the FP7 projects ALPHA and BONE is gratefully acknowledged.

\section{REFERENCES}

[1] M. Popov: The convergence of wired and wireless services delivery in access and home networks, in Proc. OFC 2010, San Diego, Mar. 2010, paper OWQ6. 
[2] A.M.J. Koonen, H.P.A. van den Boom, E. Tangdiongga, H.-D. Jung, P. Guignard: Designing in-building optical fiber networks, in Proc. OFC 2010, San Diego, Mar. 2010, paper JThA46.

[3] A.M.J. Koonen, H.P.A. van den Boom, H. Yang, C. Okonkwo, Y. Shi, S.T. Abraha, E. Ortego Martinez, E. Tangdiongga: Converged in-building networks using POF - economics and advanced techniques, in Proc. POF 2010, Yokohama, Oct. 2010.

[4] A.M.J. Koonen, M. Garcia Larrode: Radio-over-MMF techniques - Part II: Microwave to millimeter-wave systems, IEEE J. of Lightwave Technol., vol. 26, pp. 2396-2408, 2008.

[5] C.M. Okonkwo, S.T. Abraha, Y. Shi, H. Yang, H. de Waardt, E. Tangdiongga, A.M.J. Koonen: Simultaneous generation and routing of millimetre-wave signals exploiting optical frequency multiplication, in Proc. ECOC 2010, Torino, Sep. 2010, paper Th.10.B.7.

[6] A.M.J. Koonen, N.C. Tran, E. Tangdiongga: The merits of reconfigurability in WDM-TDM optical inbuilding networks, in Proc. OFC 2011, Los Angeles, Mar. 2011, paper JWA63. 